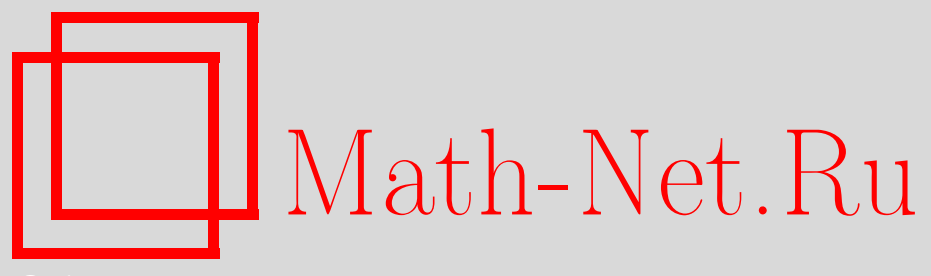

А. В. Гришин, О центре относительно свободной лиевски нильпотентной алгебры индекса 4, Матем. заметки, 2012, том 91, выпуск 1, 147-148

DOI: https://doi.org/10.4213/mzm9142

Использование Общероссийского математического портала Math-Net.Ru подразумевает, что вы прочитали и согласны с пользовательским соглашением http://www.mathnet.ru/rus/agreement

Параметры загрузки:

IP: 34.229 .108 .108

26 апреля 2023 г., 05:14:00

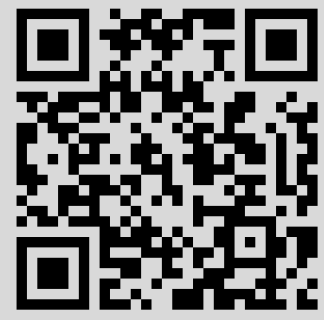




\section{О центре относительно свободной лиевски нильпотентной алгебры индекса 4}

\section{А. В. Гришин}

Пусть $F=k\left\langle 1, x_{1}, \ldots, x_{i}, \ldots\right\rangle$ - свободная ассоциативная счетнопорожденная алгебра с единицей над бесконечным полем $k$ характеристики $p>0$. Рассмотрим относительно свободную лиевски нильпотентную алгебру $F^{(\ell)}=F / T^{(\ell)}$, где $T^{(\ell)}=\left(\left[x_{1}, \ldots, x_{\ell}\right]\right)^{T}-T$-идеал, порожденный длинным коммутатором $\left[x_{1}, \ldots, x_{\ell}\right]$. При $\ell=3$ мы получаем относительно свободную алгебру Грассмана, $T$-пространства и центр в которой достаточно хорошо изучены (см. [1]-[3]). Как уже отмечалось в [4], строение $T$-пространств в $T^{(3)} / T^{(4)}$ заметно отличается от $T^{(2)} / T^{(3)}$. Однако центр алгебры $F^{(4)}$ при $p>3$ имеет достаточно хорошее описание (правда несколько более сложное, чем центр алгебры $F^{(3)}$ ).

Для простоты переменные $x_{i}$ и их образы в алгбре $F^{(\ell)}$ будем обозначать одними и теми же буквами. Напомним некоторые факты, которые используются при изучении алгебры $F^{(\ell)}$ (cм. [4]-[6]).

I. Соотношения Фробениуса. Если $p^{s} \geqslant \ell$ и $p>2$, то в алгебре $F^{(\ell)}$ справедливы соотношения

1) $\left[x_{1}^{p^{s}}, x_{2}\right]=0$;

2) $\left(x_{1}+x_{2}\right)^{p^{s}}=x_{1}^{p^{s}}+x_{2}^{p^{s}}$;

3) $\left(x_{1} x_{2}\right)^{p^{s}}=x_{1}^{p^{s}} x_{2}^{p^{s}}$.

II. Лемма Латышева. $T^{(r)} T^{(s)} \subset T^{(r+s-2)}$.

III. Лемма Воличенко. Если характеристика поля $k$ больше 3 и $\ell \geqslant 4$, mо $T^{(\ell-2)} T^{(3)} \subset$ $T^{(\ell)}$, в частности $T^{(3)} T^{(2)} \subset T^{(4)}$.

IV. $T^{(\ell-1)} \underbrace{T^{(2)} \ldots T^{(2)}}_{n} \not \subset T^{(\ell)}$ при нечетном $\ell$ и любом $n$.

ЗАмечание 1. Из леммы Воличенко следует, что при $\ell=4 \bar{T}^{(3)}=T^{(3)} / T^{(4)}$ лежит в центре алгебры $F^{(4)}$.

Отметим, что соотношения Фробениуса зависят от двух переменных и, следовательно, имеют место также и в относительно свободной альтернативной лиевски нильпотентной алгебре.

В теории T-пространств (линейных подпространств относительно свободных алгебр, замкнутых относительно эндоморфизмов этих алгебр) важную роль играет $T$-пространство $W_{p}$ в алгебре $F^{(\ell)}$, порожденное всеми $p$-словами, т.е. одночленами, в которые каждая переменная входит с кратностью $p$. Отметим, что $T$-пространство $W_{p}$ является подалгеброй в алгебре $F^{(\ell)}$. Одним из важных применений соотношений Фробениуса является (см. [4])

Теорема 1. Если $p \geqslant \ell>2$, то $W_{p}$ раскладывается в прямую сумму $T$-пространств

$$
W_{p}=D_{p} \oplus C D_{p},
$$

где $D_{p}=\left\{x_{1}^{p}\right\}^{T}$ - T-пространство, порожденное $p$-й степенъю переменной (подалгебра, изоморфная алгебре коммутативных многочленов от счетного набора переменных), a $C D_{p}$ - пересечение $W_{p}$ с коммутатором алгебры $F^{(\ell)}$, являющееся ненильпотентным нильрадикалом $W_{p}$, на котором выполнено тождество $x_{1}^{p}=0$.

(C) А. В. Гришин, 2012 
Всюду ниже $\ell=4$ и $p>3$. Согласно [3] центр алгебры $F^{(3)}$ совпадает с образом алгебры $W_{p}$ в алгебре $F^{(3)}$. Поэтому достаточно проанализировать какие элементы из $W_{p}=D_{p} \oplus C D_{p}$ лежат в центре алгебры $F^{(4)}$. Ясно, что $D_{p}$ лежит в центре. Алгебра $C D_{p}$ по модулю $\bar{T}^{(3)}$ описана в [2]. Она (с точностью до $\bar{T}^{(3)}$ ) состоит из всевозможных линейных комбинаций выражений вида

$$
c_{1} \ldots c_{m} d^{p}
$$

где $c_{i}$ - многочлены вида $\left[x_{1}, x_{2}\right] x_{1}^{r_{1}} x_{2}^{r_{2}}$ от непересекающихся множеств переменных, $d-$ произвольный одночлен, в который не входят переменные из $c_{1}, \ldots, c_{m}$.

Если $m>1$, то из леммы Воличенко сразу следует, что выражение (1) лежит в центре. Покажем, что этим все и исчерпывается. Другими словами, если в некоторой линейной комбинации выражений (1) нетривиальным образом присутствует слагаемое с $m=1$, то эта линейная комбинация не лежит в центре алгебры $F^{(4)}$. Допустим, что некоторый полиоднородный элемент вида $\left[x_{1}, x_{2}\right] x_{1}^{r_{1}} x_{2}^{r_{2}} d^{p}+\cdots$ централен. Полагая все переменные кроме $x_{1}$ и $x_{2}$ равными 1 , получаем, что элемент $\left[x_{1}, x_{2}\right] x_{1}^{r_{1}} x_{2}^{r_{2}}$ централен. Применяя подстановку $x_{1} \mapsto x_{1}+1, x_{2} \mapsto x_{2}+1$ и выделяя (что всегда возможно в силу бесконечности поля $k$ ) полиоднородную компоненту типа $(1,1)$, получаем, что $\left[x_{1}, x_{2}\right]$ лежит в центре алгебры $F^{(4)}$. Но это невозможно, так как $\left[x_{1}, x_{2}, x_{3}\right]$ не лежит в $T^{(4)}$. Отсюда следует

Теорема 2. При $p>3$ иентр алгебры $F^{(4)}$ совпадает с

$$
D_{p} \oplus\left(C D_{p}^{2}+\bar{T}^{(3)}\right) .
$$

ЗАмЕчАниЕ 2. Представляет значительный интерес вопрос об описании центра алгебры $F^{(\ell)}$ при $\ell>4$.

\section{СПИСОК ЦИТИРОВАННОЙ ЛИТЕРАТУРЫ}

[1] А. В. Гришин, Л. М. Цыбуля, Матем. сб., 200:9 (2009), 41-80. [2] А. В. Гришин, Л. М. Цыбуля, Фундамент. и прикл. матем., 15:8 (2009), 3-93. [3] А. В. Гришин, УМН, 65:4 (2010), 191-192. [4] А. В. Гришин, "О Т-пространствах в относительно свободной двупорожденной лиевски нильпотентной алгебре индекса 4", Фундамент. и прикл. матем. (в печати). [5] В. Н. Латышев, Сиб. матем. журн., 6 (1965), 1432-1434. [6] И. Б. Воличенко, T-идеал, порожденный элементом $\left[x_{1}, x_{2}, x_{3}, x_{4}\right]$, Препринт, Институт математики АН БССР, Минск, 1978.

\section{А. В. Гришин}

Поступило

Московский педагогический государственный университет

22.04 .2011

E-mail: grishinaleksandr@yandex.ru 